\title{
Nuevas tendencias del Derecho Constitucional
}

\author{
New trends of Constitutional Law \\ Alan Felipe Salazar Mujica ${ }^{1, \risingdotseq-}$ \\ 1 Facultad de Derecho y Ciencia Política Universidad Andina del Cusco, Cusco, Perú. \\ ” asalazar@uandina.edu.pe
}

\begin{abstract}
Resumen
En el presente artículo el autor realizó un repaso de los principales aspectos referidos al constitucionalismo y la Teoría Constitucional, con el fin de identificar cuáles son las nuevas tendencias que vienen alimentando el derecho constitucional de nuestros días, analizando la teoría del principialismo, el paradigma de los derechos humanos, la técnica de la ponderación, la corriente del neoconstitucionalismo y la influencia del control de convencionalidad y del Tribunal Supremo de los Estados Unidos de América.
\end{abstract}

Palabras Clave: Derecho Constitucional, constitucionalismo, principialismo, neoconstitucionalismo

\begin{abstract}
In this article the author makes an overview of the main aspects related to constitutionalism and Constitutional Theory, in order to discuss what the new trends are fueling the constitutional right of our day, analyzing the theory of principlism, the phenomenon of human rights, the weighting technique, the current neoconstitutionalism and the influence of conventionality control and the Supreme Court of the United States of America.

Keywords: Constitutional law, constitutionalism, principlism, neoconstitutionalism
\end{abstract}

Citar como: Salazar, AF. (2019), Nuevas tendencias del derecho constitucional. Rev Yachay, 8(1), 601-606.

Recibido: 16-09-2019; Aceptado 08-12-2019; Publicado 12-12-2019

\section{Introducción}

La noción de que el Derecho Constitucional, hoy en día, ha adquirido un papel protagónico en el desarrollo del Derecho, así como, en el funcionamiento del sistema de normas, está presente de alguna manera en todos los integrantes de la comunidad jurídica; desde los estudiantes de pre y posgrado, pasando por los abogados y operadores de los órganos judiciales, hasta los magistrados, académicos y iusfilósofos, poseen en algunos casos una idea bastante básica y en otros una posición debidamente analizada y argumentada sobre la esencialidad y fundamentalidad de la Constitución, pero todos parecen ser consientes de la importancia de nuestra norma superior.

En ese panorama, el presente ensayo pretende hacer un repaso sobre algunas tendencias que en la actualidad, y en los últimos tiempos, vienen alimentando la idea de constitución y principalmente su forma de aplicación y el porqué de su trascendencia como instrumento político y jurídico posibilitador de la convivencia social. Para ello, repasaremos de manera breve aquellas teorías, hechos históricos y desarrollos normativos y jurisprudenciales que han sido y son muy influyentes en el desarrollo de la Teoría de la Constitución y el Derecho Constitucional. Empezaremos con un acercamiento a la Historia de la Constitución y la Teoría Constitucional.

\section{Constitucionalismo}

El término Constitucionalismo es otra de esas palabras jurídicas que tienen la característica de la polisemia, lo que significa que esta posee varios sentidos o acepciones. Dentro de lo jurídico encontramos que un primer sentido está referido a aquellos movimientos constitucionalistas que surgieron con las revoluciones francesa y estadounidense (Constitucionalismo Crítico, 2012), pero que podrían 
incluir, además, momentos históricos anteriores y posteriores que ayudaron a construir el orden constitucional. Un segundo sentido tendría que ver con equiparar el constitucionalismo con una técnica o ideología, las dos orientadas a asegurar la limitación del poder y la garantía de los derechos a través de la constitución (Constitucionalismo Crítico, 2012) Ambas acepciones son las que más nos interesan en el presente estudio, la primera como desarrollo histórico y la segunda en el ámbito de las nuevas tendencias.

Haciendo un repaso de la historia de la Constitución, encontramos que, en la edad media, entre 930 y 1262 , el primer país en tener lo que en el mundo moderno es una Constitución fue Islandia, el que contó con un régimen político que poseía Constitución, Parlamento y Tribunales de Justicia. Claro que no fue muy importante debido a su lejanía y porque no tuvo proyección histórica a nivel mundial (Sardón, 1994).

Sin embargo, si hay un país que mediante sus hechos históricos y documentos jurídicos ayudó significativamente a construir la idea de Constitución, este fue Inglaterra. En primer término, tenemos la Carta Magna de 1215, documento que aunque no tiene nada que se parezca a una declaración de derechos del hombre, su importancia radica en que por primera vez logró fijar ciertos límites a la autoridad anteriormente ilimitada del Rey, introduciendo la noción de que la ley estaba por encima de la voluntad del monarca. Posteriormente, un conato de declaración de derechos ciudadanos aparece con el Bill of Rights, que se dicta como consecuencia de la revolución gloriosa en 1689, la que fue la culminación de una larga etapa de convulsión social, que se extendió a lo largo de todo el siglo XVII en Inglaterra. Pero va a ser con el Reform Bill (Documento de Reforma) otorgado en 1832, con el que ya se puede hablar de una democracia auténtica en Gran Bretaña, pues la Cámara de los Comunes, que son elegidos por los ciudadanos, adquiere verdaderas facultades de gobierno. De esta manera, podemos decir que cuando hablamos de la Constitución inglesa nos estamos refiriendo al conjunto de estos documentos (Carta Magna, Bill of Rights, Reform Bill, Parlamanet Act, y otros), junto con las costumbres, tradiciones y usos políticos que se han ido decantando con el tiempo; por lo que podemos decir que está principalmente escrita en el corazón de los ciudadanos ingleses (Sardón, 1994).

El desarrollo constitucional tendrá su más importante momento en el siglo XVIII, con la aparición de la Constitución de los Estados Unidos, hito histórico que es considerado por, prácticamente, todos los estudiosos del Derecho Constitucional como uno de los momentos fundamentales del constitucionalismo. ${ }^{1}$ La suscribieron en 1787, trece ex-colonias inglesas en América del Norte, luego de independizarse de Inglaterra en 1776. Se dice que los constituyentes norteamericanos estuvieron inspirados por "El Federalista", una publicación escrita principalmente por James Madison, quien también fue el más influyente en la redacción de la Constitución, cuya versión original establecía en pocos artículos la estructura del gobierno federal añadiéndosele posteriormente una serie de enmiendas, de las cuales las más importantes son las primeras diez, las que conforman el Bill of Rights de 1789 por el que se establecen los derechos ciudadanos (Sardón, 1994).

Sea como proceso histórico a favor de la constitución o como ideología de control del poder, necesariamente el término alude a un fenómeno relacionado al Estado, y en especial al Estado democrático. Como fundamento de esto tenemos que la idea moderna de democracia está íntimamente vinculada a las Constituciones, teniendo incluso como principal forma de evaluar la calidad democrática de los Estados el respeto a las normas constitucionales (Sardón, 1994).

\section{Teoría de la Constitución}

La noción de Constitución está ligada a la de la existencia del Estado, siendo este documento el que posibilita que una sociedad se constituya como tal. Uno de los numerosos autores que parece tener muy claro esta idea es Zarini, quien nos dice que "desde el momento en que se es Estado, este se encuentra 'constituido' de alguna manera, y ese estar constituido es contar con una Constitución” (Zarini, citado por García, 2011).

Por otra parte, tenemos que Constitución es el nombre que recibe la norma jurídica de más alta jerarquía o importancia, tanto en el sentido formal como material, de un ordenamiento que, por lo general, se trata de una norma escrita y de difícil modificación (Constitucionalismo Crítico, 2012).

Así mismo, podemos considerar que la Constitución es un instrumento político-jurídico que contiene un conjunto de valores, principios, normas y prácticas básicas destinadas a legitimar, modelar, organizar, regular e impulsar un tipo de sociedad política (García, 2011).

\footnotetext{
${ }^{1}$ Los distintos autores consideran a la Constitución de los Estados Unidos como parte fundamental de la historia constitucional, consignándola como un hecho decisivo en alguno de los constitucionalismos históricos, dependiendo del autor.
} 
Podemos mencionar que de forma unánime se ha establecido que la Constitución tiene dos aspectos: uno dogmático, en el que se señalan los derechos ciudadanos frente al Estado; y otro orgánico, en el que se determina la organización y la estructura del sistema de gobierno. Considerando además que la plena vigencia de ambos en un país configura lo que denominamos: "Estado Constitucional".

Tanto la parte dogmática como la parte orgánica de una Constitución son fundamentales; sin embargo, en caso de ser necesario establecer cuál de ellos es más importante para que un gobierno clasifique como democrático, podemos decir que un gobierno que no respeta el sistema de gobierno constitucional conforma una dictadura autoritaria; en cambio uno que no respeta tampoco los derechos ciudadanos o humanos, conforma una dictadura totalitaria (Sardón, 1994). Si tendríamos que distinguir el aspecto más importante de una Constitución, el contenido dogmático terminaría teniendo mayor trascendencia política, ya que el respeto a los derechos ciudadanos o humanos es la piedra de toque fundamental, desde el punto de vista constitucional, para evaluar la calidad democrática de un gobierno.

El contenido dogmatico de las constituciones ha ido cambiando a lo largo de la edad moderna, vemos que este estuvo constituido originalmente, por lo derechos civiles que el Estado debía reconocer a los ciudadanos, para que posteriormente adquirieran un contenido adicional, preocupándose por el bienestar económico y social de las personas (Sardón, 1994).

\section{Principialismo}

Fue el filósofo del derecho Ronald Dworkin, quien por primera vez planteó un modelo de principios en contraposición al modelo de reglas del sistema jurídico, planteado por autores como Kelsen o Hart, vigente hasta nuestros días, pero con mayor auge en la primera mitad del siglo XX (Alexy, 2006).

Según este enfoque principialista planteado por Dworkin, el Derecho no sólo está integrado por reglas sino también por principios, a los que se recurre ante un caso difícil, como sería la ausencia de una regla positiva que resuelva dicho caso. Siempre hay una y solo una respuesta correcta en el derecho y el juez debe aspirar a ser un "Hércules" equipado de una serie de habilidades y capacidades que lo harían capaz de encontrarla (Alexy. 2006).

En ese mismo sentido, el profesor alemán Robert Alexy, propone un enfoque similar, pero con ciertas y marcadas diferencias; nos dice que el Derecho está integrado por normas las cuales se dividen en reglas y principios, que no se puede esperar una sola repuesta correcta en los casos difíciles, porque los jueces no disponen de tiempo ni conocimiento ilimitado y que si dos principios entran en colisión se recurre a la ponderación. Ponderar es dar un peso a cada principio en relación al caso concreto, para preferir uno en lugar del otro (Alexy, 2006).

Si existen coincidencias entre Dworkin y Alexy, sin duda, una de ellas es que ambos ayudan a construir los criterios de distinción entre reglas y principios, aspecto sustancial de ambas teorías. Así encontramos que:

En cuanto a los principios, la obligación abstracta de aplicar la consecuencia jurídica de estos puede tornarse no obligatoria en casos concretos y el derecho contenido en el principio puede ser afectado u optimizado en algún grado; por tanto, los principios son graduables y optimizables. Además de ello, los principios entran en colisión en casos concretos, es decir cuando la satisfacción de un principio implica la afectación del otro y dicha colisión se resuelve con la precedencia de uno sobre el otro en virtud de su peso según el caso concreto; por tanto, los principios son ponderables (Alexy, 2006).

Por otra parte, acerca de las reglas, tenemos que la obligación abstracta de aplicar la consecuencia jurídica de estas, nunca puede tornarse no obligatoria en casos concretos; en consecuencia, las reglas son de aplicación todo o nada; por tanto, las reglas no se gradúan ni optimizan. Cuando las reglas entran en contradicción (incompatibilidad), estos casos se resuelven por el criterio de ley superior o ley posterior y la regla que no prima queda invalidada, y si se presenta una excepción se resuelve por el criterio de especialidad; por tanto, en caso de contradicción entre reglas no hay ponderación (Alexy, 2006).

\section{Derechos fundamentales}

En la actualidad, los derechos humanos se han convertido en un pilar fundamental para el mantenimiento de un sistema democrático y de un verdadero estado de derecho (Novak y Namihas, 2004). Según Constitucionalismo Crítico, autores del Diccionario de Derecho Constitucional Contemporáneo, tenemos que la expresión "derechos humanos" refiere al conjunto de atributos y facultades propios de la persona humana y por tanto han sido establecidos por los distintos ordenamientos jurídicos alrededor del mundo, implicando su defensa y protección (Constitucionalismo Crítico, 2012). 
Según John Locke los derechos civiles son tres: el derecho a la vida, el derecho a la libertad y el derecho a la propiedad (Sardón, 1994). A ellos de forma necesaria se debe aumentar los derechos políticos como el derecho al sufragio y el derecho a ocupar puestos de gobierno. Finalmente, la historia ha reconocido los derechos sociales y económicos, cuyo desarrollo y protección no posee acuerdo unánime en la comunidad académica y política.

Por otra parte, vemos que los derechos humanos son derechos básicos del ser humano, que engloban la totalidad de sus dimensiones: aspecto físico, aspecto psicológico y aspecto social; abarcando una concepción integral del ser humano, lo que nos permite considerar un catálogo abierto y extenso de atributos que podrían ser reconocidos o reivindicados como derechos fundamentales, acercándonos a una concepción profundamente humanista y abierta del hombre, reemplazando la tradicional visión patrimonialista o civil que consideraba un catalogo de derechos positivos cerrados. Así mismo, podemos apuntar algunas características doctrinarias de los derechos humanos, como son: su universalidad, su indivisibilidad, su carácter igualitario y su carácter fundamental (Sardón, 1994).

Además del sustento teórico o filosófico de los derechos humanos, encontramos que hoy en día estos se encuentran regulados o consagrados, no solo en las constituciones o normas de los sistemas jurídicos estatales, sino principalmente en las normas internacionales, existiendo numerosos tratados y declaraciones elaboradas en el ámbito de ciertas organizaciones internacionales que consagran derechos fundamentales de las personas e incluso, los mecanismos para su protección (Novak y Namihas, 2004).

Luego de 1948 se inició una configuración de catálogos internacionales y la proliferación de listados extensos de derechos positivos internacionales, de esta forma se advierten declaraciones, pactos y convenciones, en lo global y regional, todos ellos expresión del "evangelio" de la cultura contemporánea; e incluso dándose una extensión creciente en la identificación de los distintos derechos humanos, encontrando como elemento definitivo la progresividad (Agudelo, 2015).

Desde la teoría jurídica, la tendencia actual es la de darle a los derechos fundamentales la categoría de normas principio, esto sin duda ayuda a solucionar los posibles problemas que ocurren en su aplicación.

\section{Ponderación}

Uno de los aspectos principales de la llamada Teoría de la Argumentación Jurídica es sin duda la aplicación del derecho en los casos concretos. Agregando a ello, y tomando en cuenta que el argumentacionismo considera la misma clasificación de normas jurídicas que el principialismo (principios y reglas), es pertinente expresar que la ponderación es sin duda, parte fundamental de las nuevas tendencias del Derecho constitucional, más aún cuando Alexy ha desarrollado este principio en su propuesta teórica sobre los derechos fundamentales.

Según Bernal, la ponderación es la forma en que se aplican los principios jurídicos, es decir, las normas que tienen la estructura de mandatos de optimización, pues determinan que algo sea realizado en la mayor medida posible, dentro de las posibilidades jurídicas y reales existentes (Bernal, 2003). Cuando se pretende aplicar el mandato de optimización previsto en el principio respectivo, se tiene que generar una medida pública o dictar una sentencia, en la que se aplique dicho principio. Sin embargo, en el proceso de optimización de un principio se encuentran obstáculos relativos a las posibilidades jurídicas y fácticas.

La ponderación es otro método estructural, como también lo es el denominado silogismo jurídico. Es utilizable en los denominados casos difíciles, relativos a colisiones de principios sobre derechos fundamentales. Consiste en el proceso de asignación de pesos a los principios en conflicto, para preferir aquel que haya obtenido mayor peso de importancia (Balancing). La ponderación implica - en un caso concreto de colisión de principios - la preferencia por el principio de mayor importancia y la afectación de otro principio que se considere de menor importancia. La ponderación es un procedimiento racional (pero limitado) que contribuye a la solución de conflictos entre principios, pero no ofrece resultados definitivos y exentos de alguna subjetividad. La ponderación dota de coherencia lógica al razonamiento jurídico, pero no en sentido absoluto, porque sus resultados no siempre son unívocos (Bernal, 2003).

Es Robert Alexy, citado por Bernal (2003), quien con mayor claridad y precisión ha expuesto la estructura de la ponderación. De acuerdo con él, los tres elementos que forman la estructura de la ponderación son:

a) La ley de la ponderación: según la cual "cuanto mayor es el grado de la no satisfacción o de afectación de uno de los principios, tanto mayor debe ser la importancia de la satisfacción del otro". A su vez esta ley, se divide en tres pasos, a saber: definir el grado de la no satisfacción o afectación de uno de los principios; definir la importancia de la satisfacción del principio que juega en sentido contrario; y definir si la importancia de la satisfacción del principio que juega en contrario justifica la afectación o la no satisfacción del otro. 
b) La fórmula del peso: expresa que el peso del principio P1 en relación con el principio P2 en las circunstancias de un caso concreto, resulta del cociente entre el producto de la afectación del principio P1 en concreto, su peso abstracto y la seguridad de las premisas empíricas relativas a su afectación, por una parte; y el producto de la afectación del principio P2 en concreto, su peso abstracto y la seguridad de las premisas empíricas relativas a su afectación, por otra.

c) Las cargas de argumentación: estas operan cuando existe un empate entre los valores que resultan de la aplicación de la fórmula del peso, es decir, cuando los pesos de los principios son idénticos. En este punto Bernal Pulido considera que Alexy, parece defender dos posiciones, pues en su obra "Teoría de los derechos fundamentales" expone que la carga argumentativa será a favor de la libertad jurídica y la igualdad jurídica; mientras que, en otro trabajo, escrito quince años después, un empate jugaría a favor del legislador y el principio democrático en el que se funda la competencia del parlamento.

\section{Neoconstitucionalismo}

Sin duda, el fenómeno jurídico-constitucional más difundido en la actualidad y que además ha generado una serie de debates académicos y filosóficos, es el neoconstitucionalismo. Esta corriente, que cada vez cuenta con la simpatía y el respaldo de un mayor número de colegas, se muestra como una perspectiva anti-iuspositivista, lo que también le ha significado acumular un gran número de detractores.

De acuerdo con Miguel Carbonell, si quisiéramos responder preguntas como ¿si en verdad existe algo nuevo en el neoconstitucionalismo? o ¿de qué hablamos cuando hablamos de neoconstitucionalismo?, hay por lo menos tres niveles de análisis que es conveniente examinar (Carbonell, 2010).

a) El primer nivel tiene que ver con los textos constitucionales, pues se dice que el neoconstitucionalismo pretende explicar un conjunto de textos constitucionales aparecidos posteriormente a la segunda guerra mundial y que contienen amplios catálogos de derechos fundamentales, además de normas que condicionan la actuación del estado por medio de la ordenación de ciertos fines y objetivos.

b) Como una consecuencia de la aparición de los textos constitucionales tenemos a las prácticas jurisprudenciales, pues encontraremos que estas han ido cambiando en muchos tribunales y cortes en materia constitucional, donde los jueces vienen realizando su función bajo parámetros interpretativos nuevos, propios del principialismo; donde entran en juego la ponderación, la proporcionalidad, la razonabilidad, la maximización de los efectos normativos de los derechos fundamentales, entre otros.

c) Finalmente, encontramos a los desarrollos teóricos novedosos, que parten de los textos constitucionales y el desarrollo jurisprudencial, contribuyendo de esa manera a entender de mejor manera el fenómeno jurídico, e incluso más allá de eso alcanzando el nivel de crearlo.

Por su parte, la profesora italiana Susanna Pozzolo, denominó como neoconstitucionalismo a una corriente integrada por un grupo de iusfilósofos que comparten un modo muy particular de acercarse al derecho y que defienden la tesis sobre la especificidad de la interpretación constitucional, según ella estos son: Dworkin, Alexy, Zagrebelsky y Nino en alguna medida.

Pozzolo acepta que estos autores no se reconocen dentro de una corriente unitaria específica, pero considera que a favor de su planteamiento existen una serie de rasgos que permiten agruparlos de esta manera (Pozzolo, 1997). Para la profesora de la Universidad de Génova, las características peculiares que posibilitan que los mencionados iusfilósofos sean agrupados en una sola corriente, se pueden formular de la siguiente manera:

i. Principios versus normas, referido a que el ordenamiento jurídico no solo está constituido por normas sino también por principios;

ii. Ponderación versus subsunción, referido al peculiar método interpretativo/aplicativo que requieren los principios;

iii. Constitución versus independencia del legislador, con la que se expresa la tesis que sostiene una penetración general del texto constitucional; y

iv. Jueces versus libertad del legislador, referida a la expresión de una tesis favorable a la interpretación creativa de la jurisprudencia (Pozzolo, 1997).

\section{Derecho Internacional y Derecho Comparado}

Sin tener el impacto práctico y teórico de las tendencias en sentido estricto, consideramos que no podemos dejar de mencionar algunos sistemas, órganos y técnicas jurídicas que en diferente medida participan de la pauta que guía el desarrollo del Derecho Constitucional en nuestros tiempos. En ese sentido tenemos: 
a) El control de convencionalidad: es innegable la influencia que la Corte Interamericana ha venido ejerciendo en la evolución y transformación del derecho constitucional de los Estados latinoamericanos mediante su jurisprudencia, tal como se puede ver tras el análisis de diferentes casos emblemáticos tales como: Almonacid Arellano contra Chile, Bulacio vs. Argentina, "La Última Tentación de Cristo", Los 19 comerciantes vs. Colombia, Barrios Altos vs. Perú, entre muchos otros.

b) La Corte Suprema de Justicia de los Estados Unidos de América: desde sus inicios con el naciente Estado norteamericano que llegaría a convertirse en la primera potencia mundial, la influencia de sus decisiones ha trascendido al derecho angloamericano, realizando aportes importantes al derecho constitucional. Desde el emblemático caso Marbury vs. Madison, pasando por otros de trascendencia como Brown vs. el Consejo de Educación y Aaron vs. Cooper, hasta más recientes como Lawrence vs. Texas y recientemente el caso Obergefell contra Hodges.

\section{Conclusiones}

Primera. Consideramos que el desarrollo de la Constitución y del Derecho Constitucional, es un proceso histórico, político y jurídico que ha evolucionado a través de los tiempos, teniendo sus mayores momentos de madurez a partir del siglo XVIII.

Segunda. Podemos expresar que la Constitución reúne como parte de su esencia el fenómeno de la formación del Estado moderno, con la dinámica política y la normatividad jurídica, siendo trascedente en estos tres ámbitos, haciendo posible además la materialización de la ideología democrática.

Tercera. Las ideas del principialismo sobre la división de las normas jurídicas en principios y reglas, han otorgado sustento y fuerza normativa a la Constitución, situación que hasta ese momento estaba más desarrollada en el ámbito político.

Cuarta. El paradigma de los derechos humanos también encuentra mayor fuerza normativa a razón del principialismo y sobre todo por su inclusión en los textos constitucionales.

Quinta. La ponderación como forma de aplicación de los principios constitucionales se consolida como un eficaz instrumento para la solución de los llamados casos difíciles, de acuerdo a la nueva concepción de la constitución como una norma jurídica directamente aplicable.

Sexta. El neoconstitucionalismo es una tendencia o corriente de pensamiento que se alimenta de diferentes estudios jurídicos, principalmente en materia constitucional, y que logra organizar y sistematizar una serie de aspectos que sustentan la dinámica del derecho contemporáneo.

Séptima. Además de los aportes teóricos y jurisprudenciales de las tendencias principialista, argumentacionista y neoconstitucionalista, son importantes también el desarrollo y la influencia de la Corte Interamericana mediante el control de convencionalidad, y de la Corte Suprema de Justicia de los Estados Unidos, mediante sus casos emblemáticos.

\section{Bibliografía}

Agudelo, M. (2015). Cine y Derechos Humanos. Una aventura filmica. Medellín: UNAULA.

Alexy, R. (2006). Derecho y razón práctica. México D.F.: Fontamara.

Bernal, C. (2003). Estructura y Límites de la ponderación. Revista DOXA $\mathrm{N}^{\circ} 26$, Universidad de Alicante, Espagrafic. http://publicaciones.ua.es/filespubli/pd f/02148676RD49949854.pdf. Consultado el 24 de noviembre de 2013.

Constitucionalismo Crítico. (2012). Diccionario de Derecho Constitucional Contemporáneo. Lima: Gaceta Jurídica.
Novak, F., y Namihas, S. (2004). Derecho Internacional de los Derechos Humanos. Lima: Academia de la Magistratura.

García, V. (2011). Estudio introductorio a la Constitución de 1993. en TC Gaceta Constitucional. La Constitución Política del Perú. Lima: Gaceta Jurídica.

Pozzolo, S. (1997). Neoconstitucionalismo y especificidad de la interpretación constitucional. Revista DOXA No 21II, Universidad de Alicante, Espagrafic. https://rua.ua.es/dspace/bitstream/1004 5/10369/1/doxa21-225.pdf

Consultado el 7 de agosto de 2016.
Sardón de Taboada, J. L. (1994). Estado, Política y Gobierno. Lima: Universidad del Pacífico.

VV.AA. (2019). Derecho, Constitución y Cine. Salazar, A. (director). Cusco. Red Iberoamericana de Cine y Derecho, Universidad Andina del Cusco: Dirección de Bibliotecas y Editorial Universitaria.

VV.AA. (2010). El canon neoconstitucional. Carbonell, M. / García J., L. (editores). Madrid. Inst. Investigaciones JurídicasUNAM: Trotta. 